\title{
Relative validity of a FFQ in measuring carbohydrate fractions, dietary glycaemic index and load: exploring the effects of subject characteristics
}

\author{
Niina E. Kaartinen ${ }^{1 *}$, Heli Tapanainen ${ }^{2}$, Liisa M. Valsta ${ }^{2} \dagger$, Minna E. Similä ${ }^{1}$, Heli Reinivuo ${ }^{2}$, \\ Tommi Korhonen $^{2}$, Kennet Harald ${ }^{1}$, Johan G. Eriksson ${ }^{1,3,4,5,6}$, Markku Peltonen ${ }^{1}$ and Satu Männistö ${ }^{1}$ \\ ${ }^{1}$ Department of Chronic Disease Prevention, National Institute for Health and Welfare, PO Box 30, FI-OO271 Helsinki, \\ Finland \\ ${ }^{2}$ Department of Lifestyle and Participation, National Institute for Health and Welfare, PO Box 30, FI-OO271 Helsinki, \\ Finland \\ ${ }^{3}$ Department of General Practice and Primary Health Care, University of Helsinki, PO Box 20, FI-OOO14 Helsinki, Finland \\ ${ }^{4}$ Vasa Central Hospital, Vasa, Finland \\ ${ }^{5}$ Unit of General Practice, Helsinki University Hospital, Helsinki, Finland \\ ${ }^{6}$ Folkhälsan Research Centre, Helsinki, Finland \\ (Submitted 18 January 2011 - Final revision received 17 May 2011 - Accepted 5 July 2011 - First published online 8 September 2011)
}

\section{Abstract}

FFQ require validation as part of epidemiological research of diet-disease relationships. Studies exploring associations between carbohydrate type and chronic diseases are rapidly increasing, but information on the validity of carbohydrate fractions, dietary glycaemic index (GI) and the glycaemic load (GL) estimated by FFQ is scarce. Likewise, the effects of subject characteristics on FFQ validity have been poorly documented. The present study evaluates the relative validity of an 131-item FFQ in relation to two $3 \mathrm{~d}$ food records (FR) performed 6 months apart focusing on the intake of carbohydrate fractions, dietary GI and the GL. Furthermore, we assessed the extent to which subjects' age, education and BMI explain differences between these methods. The study sample comprised 218 men and 292 women aged 25-74 years participating in a large population-based survey in Finland. Energy-adjusted Spearman's rank correlations ranged from 0.27 (sugars) to 0.70 (lactose) for men and from 0.37 (sugars) to 0.69 (lactose) for women. On average, $73 \%$ of the subjects were categorised into the same or adjacent distribution quintile based on the two methods. In general, the FFQ overestimated the intakes compared with FR. Especially in women, FFQ validity for some nutrients was associated with the level of intake, subjects' age and, to a lesser extent, education but not BMI. In conclusion, the FFQ appears to be reasonably valid in the assessment of carbohydrate exposure variables, but the findings show a need for adjustment of diet-disease relationships for subjects' age and education.

Key words: FFQ: Validity: Carbohydrates: Glycaemic index

In recent years, the potential importance of carbohydrate type in healthy diets has been repeatedly emphasised ${ }^{(1,2)}$. The effects of different carbohydrate fractions, such as fibre, starch and sugars, as well as dietary glycaemic index (GI) and the glycaemic load (GL) on various health outcomes have been increasingly studied ${ }^{(3-5)}$. The validity of dietary assessment methods in measuring different carbohydrate fractions beyond total carbohydrate and dietary fibre, however, seems to be poorly documented. For instance, FFQ validation studies have rarely included results for several carbohydrate fraction intakes, dietary GI and the $\mathrm{GL}^{(6-11)}$.
Due to feasibility and low cost, FFQ are widely used in large-scale epidemiological studies ${ }^{(12)}$. FFQ validity testing is required to picture FFQ performance and shortcomings in measuring usual intake and ranking of subjects accordingly, which generally represents the main purpose of FFQ, including the one used in the present study. Food records (FR) are recommended as the self-reported reference method for FFQ validations and they remain commonly utilised ${ }^{(13-15)}$. In general, FFQ validation studies using FR as the reference method produce acceptable results ${ }^{(10,11,16)}$

Personal characteristics of study subjects have long been supposed to affect FFQ validity ${ }^{(17)}$. In spite of this,

Abbreviations: FR, food record; GL, glycaemic load; GI, glycaemic index.

*Corresponding author: N. E. Kaartinen, fax +358 20610 8338, email niina.kaartinen@thl.fi

† Present address: European Food Safety Authority, Data Collection and Exposure, Parma, Italy. 
little documentation exists on how personal characteristics other than sex affect FFQ performance in adult populations. In one Australian study using $12 \mathrm{~d}$ weighed FR as the reference, it has been shown that age, BMI and medical condition, but not school-leaving age, were associated with the relative FFQ validity for some nutrients, including total carbohydrate, fibre, starch and sugars ${ }^{(9)}$. In an earlier Finnish study, a slightly weaker FFQ validity among younger women (30-50 years) and overweight men and women emerged when comparing FFQ estimates with $3 \mathrm{~d} \mathrm{FR}^{(18)}$. However, no difference in correlations between high- and low-educated Midwestern Americans has been found when comparing FFQ estimates with those derived from 2 to $8 \mathrm{~d} \mathrm{FR}^{(19)}$.

The aim of the present study was to evaluate the validity of an FFQ relative to FR among Finnish adults participating in a large population-based survey focusing on the intake of various carbohydrate fractions, dietary GI and the GL. Furthermore, the aim was to explore whether nutrient intake level and subject characteristics, such as age, education and BMI, are associated with the relative FFQ validity.

\section{Methods \\ Selection of study subjects}

The base population comprised the National FINRISK 2007 Study - the eighth population-based health survey conducted in 5-year intervals to monitor chronic diseases and their risk factors in Finland ${ }^{(20)}$. The FINRISK 2007 sample ( $n$ 10000, aged 25-74 years) was drawn randomly from the Finnish population register stratified by sex, 10-year age groups and five geographical areas. Of the invited subjects, 63\% participated in the health examination in January-March 2007.

Of the FINRISK sample, $33 \%$ ( $n$ 3286) were randomised to a Finnish diet and nutrition monitoring survey (FINDIET 2007 Survey), in which, concurrently with the FINRISK health examinations, 2054 (63\%) subjects performed one $48 \mathrm{~h}$ dietary recall $\mathrm{each}^{(21)}$. Half of the interviewees ( $n$ 1039) received instructions to fill in one 3d FR in January-March ( $n$ 935, returned) and a second 3d FR in June-October 2007 ( $n$ 624, returned). All FR were entered using uniform coding decisions. Due to incompleteness and missing identification labels, 606 subjects completed two 3 d FR. Further exclusions were made due to FR classified as unreliable during the data entry ( $n$ 45) and illness affecting the diet (e.g. stomach flu) during food recording ( $n$ 6).

A second study visit of all FINRISK 2007 participants was conducted in April-June 2007 to gather detailed information on the dietary, lifestyle and genetic determinants of obesity and the metabolic syndrome ( $n$ 5024, 84\%). During this study visit, subjects filled in FFQ ( $n$ 4996) and other selfadministered questionnaires, delivered blood samples and underwent anthropometric measurements. Trained study nurses checked the completed FFQ and a nutritionist entered the data. Exclusions were made due to incompletely filled FFQ ( $n$ 74) and daily energy intake cut-offs corresponding to $0.5 \%$ at both ends of the daily energy intake distributions for men and women ( $n$ 48), resulting in $4874 \mathrm{FFQ}$.
A total number of 510 (218 men and 292 women) subjects had completed both the FFQ and two $3 \mathrm{~d}$ FR and formed the final population for the FFQ validation study. The administration sequence of the test and reference method featured two food-recording periods timed approximately 6 months apart (January-March and June-October 2007) and the FFQ administered in-between these periods (April-June 2007).

The study was conducted according to the guidelines laid down in the Declaration of Helsinki and the ethical guidelines of the National Institute for Health and Welfare. All procedures involving human subjects were approved by the Ethics Committee of the Hospital District of Helsinki and Uusimaa. Written informed consent was obtained from all subjects.

\section{FFQ}

The FFQ was an updated version of the one used in earlier studies of the National Institute for Health and Welfare, for instance the Health 2000 Survey ${ }^{(18)}$. The $48 \mathrm{~h}$ dietary recalls of the FINDIET 2007 Survey were used as the data source for the updates. These included a revision of the food list to reflect the continuously growing selection of available foods. For example, commonly eaten foods, such as grapes and milk puddings, were added. We also assigned fixed FFQitem portion sizes separately for men and women based on self-reported usual portion size information derived from $48 \mathrm{~h}$ dietary recalls.

The updated 131-item FFQ was designed to measure the habitual diet over the previous 12 months. The FFQ items were presented in twelve food groups (e.g. milk products, cereal products and fruits). The subjects were asked to indicate the average consumption frequency of each FFQ item, with the nine frequency categories ranging from never or seldom to six or more times per $\mathrm{d}$. The predefined FFQ-item portion sizes appeared as household and natural units (e.g. glass or slice) on the FFQ. The subjects were also able to report other frequently consumed foods not listed.

\section{Food records}

Subjects obtained face-to-face instruction on how to record everything they ate or drank during three consecutive days starting the day after the FINRISK health examination. To facilitate the estimation of portion sizes, a 170-item photographic picture booklet was provided ${ }^{(22)}$. The second $3 \mathrm{~d}$ FR were mailed to those subjects who returned the first. Both $3 \mathrm{~d}$ FR were returned by mail to the National Institute for Health and Welfare.

\section{Personal characteristics and anthropometric measures}

Total years of education were inquired on a self-administered questionnaire and used to classify participants into three educational levels (low, middle and high) according to birth year. This was done to adjust for the extension of the basic education system and increase of average school years over time. Body weight was measured to the nearest $0 \cdot 1 \mathrm{~kg}$ and 
height to the nearest $0.1 \mathrm{~cm}$. BMI was calculated as weight $(\mathrm{kg})$ divided by the square of height $\left(\mathrm{m}^{2}\right)$.

\section{Calculation of carbohydrate fraction intakes, dietary glycaemic index and the glycaemic load}

Daily intakes were calculated using the Finnish National Food Composition Database $\left(\text { Fineli }^{\circledR}\right)^{(23)}$. The weights of the FFQ items as a daily average were obtained by multiplying the indicated frequencies of consumption by sex-specific fixed portion sizes. The Fineli ${ }^{\circledR}$ database contains GI values assigned for FFQ items and foods consumed by the FINDIET 2007 participants $^{(24)}$. Thus, $100 \%$ of the FFQ and FR items could be linked to a GI value. The methodology for the assignment of GI values to Finnish food items has previously been described $^{(24,25)}$. Dietary GI for both the FFQ and the FR were calculated as the weighted mean of the GI values of the carbohydrate-containing foods in the diet, where weighting is based on the proportion of the total carbohydrate content provided by each food ${ }^{(26,27)}$. Dietary GL was calculated by multiplying the dietary GI value with the carbohydrate content of the diet and dividing by $100^{(28,29)}$. Thus, the GL accounts for both the quality and quantity of the carbohydrates, and two diets with comparable GI can possess different GL due to largely different carbohydrate contents.

\section{Statistical methods}

All analyses were performed separately for men and women due to sex-specific portion sizes. Means and standard deviations of the selected subject characteristics (age and BMI) and educational-level proportions, as well as nutrient intakes, dietary GI and the GL from the FFQ and FR were calculated by sex. Nutrient intakes, dietary GI and the GL were log (natural)transformed in order to satisfy the normality assumption and subsequently adjusted for each individual's energy intake by using the residual method ${ }^{(30)}$. Each of the calculated residuals was added to the mean intake of the study population according to each dietary method. The food groups contributing most to the intake of total carbohydrate, carbohydrate fractions and the dietary GL were calculated.

The Spearman rank correlation coefficients between the FFQ and FR were calculated for all crude and energy-adjusted nutrients, dietary GI and the GL. The ability of the FFQ to rank subjects according to their intakes was assessed by categorising them by sex into quintiles of intake by the two methods. The proportion of subjects classified into the same or adjacent quintile and the opposite quintile (gross misclassification) by the two methods was calculated. Gross misclassification was defined as disagreement by four quintiles. The level of agreement in intake estimates between the two dietary methods was explored by calculating the mean ratios $(\mathrm{FFQ} / \mathrm{FR} \times 100)$ and corresponding $95 \% \mathrm{CI}$ of the energy-adjusted nutrient intakes, dietary GI and GL. Values greater than 100\% indicate that at the population level, the FFQ-based intake exceeds the FR-based intake, while the opposite holds true for values $<100 \%$.
Linear regression analysis was performed to investigate whether the difference in intake estimates (FFQ - FR, dependent variable) varied across the mean of intake estimates (FFQ $+\mathrm{FR} / 2$, independent variable), as suggested by Bland \& Altman ${ }^{(31)}$. The slope of the regression line was tested for a significant deviation from zero. The slopes significantly different from zero indicate a relationship between the level of intake and the measurement error in the FFQ relative to the FR. Linear regression analyses were also used to explore whether the difference between the methods was associated with each of the studied subject characteristics (age, education and BMI). All statistical analyses were performed using the SAS statistical software package version 8.2 (SAS Institute Inc., Cary, NC, USA).

\section{Results}

The participants' mean age was 55 and 52 years (range 25-74 years), and BMI was 27.2 and $26.7 \mathrm{~kg} / \mathrm{m}^{2}$ for men and women, respectively. Of men, $36.5 \%$ and of women, $36 \cdot 7 \%$ belonged to the highest educational group, while $27 \cdot 2$ and $30 \cdot 4 \%$ belonged to the lowest educational group, respectively. The means and standard deviations of the crude (unadjusted) daily nutrient intakes, dietary GI and the GL based on the FFQ and FR for men and women are given in Table 1.

The same food groups explained over $90 \%$ of the intake in each carbohydrate fraction and dietary GL in FFQ and FR. The relative contribution of the food groups differed, however, slightly between the methods. In general, milk products, fruits and berries, and juice contributed with greater percentages in the FFQ compared with FR, whereas in the latter, cereal products, sugar and confectionery, sugar-sweetened beverages, and alcoholic beverages received more emphasis.

The crude Spearman rank correlation coefficients (Table 1) ranged from 0.32 (dietary GI) to 0.68 (lactose) for men and from 0.16 (energy) to 0.67 (lactose) for women. The energyadjusted correlations were between $0 \cdot 27$ (total sugars) and 0.70 (lactose) for men and between 0.37 (total sugars) and 0.69 (lactose) for women. Overall, nine out of eleven energy-adjusted dietary factors exceeded 0.40 in both sexes.

Based on the two methods, an average of $71 \%$ (62\% for dietary GI to $81 \%$ for lactose) for men and 65\% (58\% for energy and dietary GL to $81 \%$ for lactose) for women were correctly classified in the same or adjacent quintile of crude intakes (see Appendix). The extent of correct classification was higher for the energy-adjusted intakes, on average $73 \%$ for both men (62\% for total sugars to $83 \%$ for lactose) and women (64\% for total sugars to $83 \%$ for lactose). Gross misclassification was rare for the energy-adjusted dietary factors in both sexes: $\leq 6 \%$ for men and $\leq 4 \%$ for women.

As indicated by the mean ratios (FFQ \% of FR), the FFQ consistently overestimated the energy-adjusted intakes of the nutrients and dietary GL in both sexes compared with the FR (Table 2). The most overestimated carbohydrate fractions were fructose and lactose. The Bland-Altman analysis revealed that a growing intake level in all of the studied dietary factors, except sucrose and the dietary GI, was associated with the between-method difference in women. In men, this 
phenomenon was observed for total carbohydrate, sugars, fructose, lactose, and dietary GI and GL. Figs. 1 and 2 provide examples of the Bland-Altman analysis (fructose and sucrose).

Age was associated with the absolute between-method difference in two out of eleven dietary factors in men (lactose and dietary GL; Table 3). In women, this was observed for all studied dietary factors, except starch, lactose and insoluble dietary fibre. In men, the belonging to the middle educational group (compared with the highest) was associated with the between-method difference in total and insoluble dietary fibre estimates. In women, associations emerged between the belonging to the lowest educational group (compared with the highest) and the between-method difference in total carbohydrate, starch, lactose and dietary GL estimates. BMI did not associate with the between-method difference in any of the studied dietary factors.

\section{Discussion}

The present Finnish FFQ validation study was conducted among a sample of 25-74-year-old men and women $(n 510)$ participating in a large population-based survey ( $n$ 5024). In general, the 131-item FFQ overestimated carbohydrate fraction intakes and the dietary GL compared with the two $3 \mathrm{~d}$ FR. The ability of the FFQ to rank subjects according to relative nutrient intakes and dietary GI and GL is at an acceptable level and fairly comparable with other comprehensive FFQ used in large epidemiological studies ${ }^{(11,16)}$. The level of intake, subject age and, to a lesser extent, education, however, appear to be associated with FFQ performance for some dietary factors, especially in women.

In general, correlation coefficients are the most applied statistical procedures used to analyse the validity of $\mathrm{FFQ}^{(14,32)}$. The present results for total carbohydrate and fibre compared reasonably well with those from classical validation studies $^{(11,16)}$ and earlier studies from Finland ${ }^{(10,18)}$, showing correlation coefficients in the range of $0.42-0.64$ for total carbohydrate and $0.51-0.73$ for fibre. Moreover, subject classification into the same or adjacent quintiles was generally acceptable and gross misclassification was rare, which is relevant for the intended use of our FFQ.

Thus far, only a few large $(n \geq 100)$ validation studies have reported correlations between comprehensive FFQ and FR for carbohydrate fractions beyond total carbohydrate and fibre. Energy-adjusted Spearman's or Pearson's correlations for total starch (range 0.19-0.68) and sugars (range 0.39-0.63) have been reported in six studies each ${ }^{(6-9,11,33,34)}$. Correlations for sucrose intake ranging from 0.41 to 0.69 have been reported by one study from the $\mathrm{USA}^{(16)}$, one from $\operatorname{Australia}^{(7)}$, two from Denmark ${ }^{(35,36)}$ and three from Finland ${ }^{(10,11,18)}$. Only two studies have reported correlations for lactose intake $(0.75 \text { and } 0.72)^{(7,10)}$ and one for fructose intake $(0 \cdot 66)^{(7)}$. The present findings compare reasonably well with the earlier ones for the different carbohydrate fractions. Only total sugars and fructose showed slightly lower correlations, which may in part originate from the different 
emphasis of the food groups as sources for sugars and fructose in the two methods.

Similar to different carbohydrate fractions, information on the validity of FFQ to measure dietary GI and GL has been scarce, since only five studies have been published using either FR comprising a total of $12-28 \mathrm{~d}$ or $12-24 \mathrm{~h}$ dietary recalls as the reference ${ }^{(6,7,37-39)}$. In these studies, energyadjusted correlation coefficients in the range of $0 \cdot 40-0 \cdot 69$ for dietary GI and $0.32-0.70$ for dietary GL have been reported. Thus, the correlations in the present study are in the lower boundary of these ranges. We observed an emphasis of medium- and high-GI foods (sugar and confectionery, soft drinks and alcoholic beverages) as carbohydrate sources in the FR compared with FFQ, whereas low-GI foods (milk products, fruits and vegetables) were emphasised in the latter. This divergence may in part contribute to the present results. Overall, possible reasons for the discrepancy across studies may relate to the variability of carbohydrate sources in populations and challenges in applying GI values to foods.

In general, variation in correlation results across studies may be due to population differences. A heterogeneous population, with regard to sex and age, can magnify between-person variation in diet and thus the correlations. Furthermore, comparing validation results is challenging, since the FFQ food lists differ in length. The number of FFQ items seems to be of importance, when exploring specific carbohydrate fractions and dietary GI and GL. Detailed questionnaires $^{(11,34)}$ and questionnaires especially designed to measure carbohydrate intake ${ }^{(7,39)}$ have produced high correlations. However, FFQ with few items (e.g. $<100$ items) show good validity for total carbohydrate, sucrose and fibre, even in populations restricted by sex and age ${ }^{(16,35,36)}$.

The number of food-recording days required to represent the usual diet, including carbohydrate intake, largely depends on the within-person variation in food consumption and nutrient intake ${ }^{(12)}$. In general, higher correlations are observed, when the reference comprises several days ${ }^{(15)}$. The earlier version of the present FFQ was validated against $3 \mathrm{~d} \mathrm{FR}^{(18)}$. Compared with that study, we observed slightly better correlations for carbohydrate in both sexes, and sucrose and fibre in men. An earlier forerunner of our FFQ was validated against $14 \mathrm{~d}$ FR among 25-75-year-old women and showed poorer correlations for total carbohydrate and fibre than the present study, but better validity for lactose and sucrose ${ }^{(10)}$. Thus, it seems that total carbohydrate and fibre intakes may be well covered with few recording days, whereas specific carbohydrate fractions require more.

The time sequence and the implementation order of the two methods can also affect between-method correlations. FFQ validation studies are conducted in a variety of different time sequences and variation in the number of consecutive FR days exists ${ }^{(12,15)}$. This FFQ inquired about food consumption during the previous 12 months, and it was completed inbetween the two food-recording periods, which formed the reference. Ideally, the FFQ should represent the time scale, during which the FR are kept ${ }^{(12)}$. Unfortunately, this requirement was not completely fulfilled in the present study due to practical issues of the study protocol. 

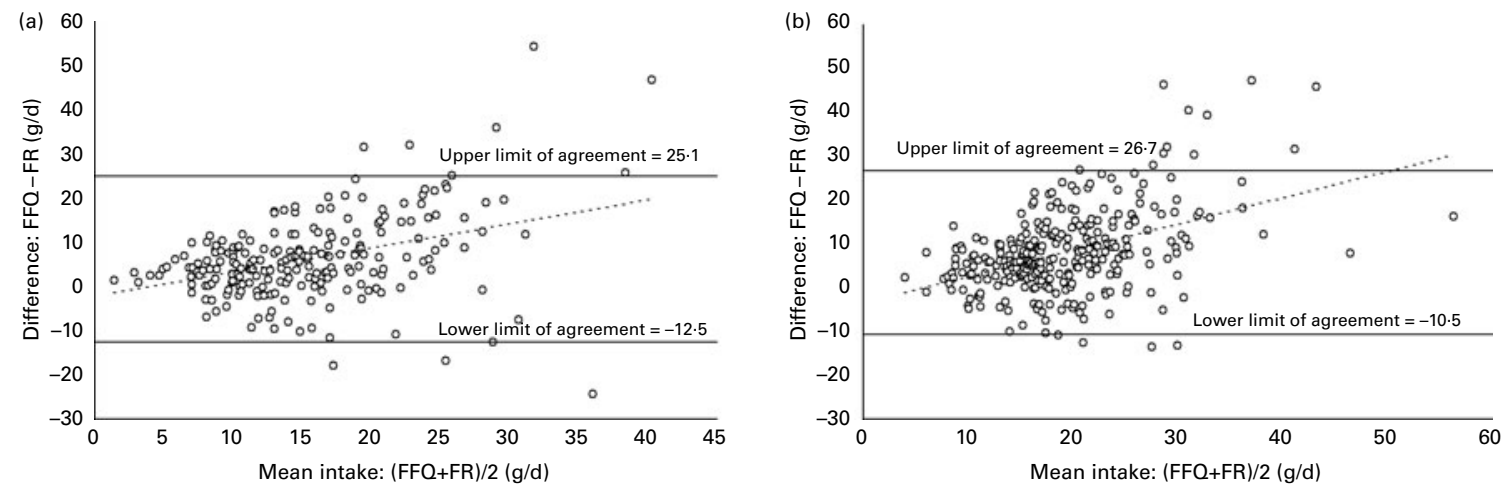

Fig. 1. Bland-Altman plots ${ }^{(31)}$ of fructose from the FFQ and two $3 d$ food records (FR) for (a) men $(n 218 ; y=-0.54 \times-2 \cdot 1, P<0.0001)$ and (b) women ( $n$ 292; $y=-0.60 x-3.5, P<0.0001)$. The linear trend $\left(\mathrm{H}_{0}\right.$ : slope $\left.=0, \alpha=0.05\right)$ and the limits of agreement are indicated. Intake values are energy-adjusted using the residual method ${ }^{(30)}$.

At the population level, FFQ commonly overestimate carbohydrate fraction intakes compared with $\mathrm{FR}^{(8,11,18,34)}$. This occurred also in the present study and might relate to general difficulties in self-reported dietary assessment. Bias may originate from the relative overestimation of foods (and related nutrients) perceived as socially desirable and energy (macronutrient) under-reporting. The latter consistently associates with higher BMI and other subject characteristics such as female sex and old age ${ }^{(40)}$. The extent to which energy under-reporting occurs is, however, likely to be populationand method-specific ${ }^{(41)}$. In estimated FR, the median prevalence of energy under-reporting is $30 \%{ }^{(40)}$. Thus, the observed overestimation of the FFQ may in part arise from underreporting in FR, but the in-depth analysis of this is beyond the scope of the present study.

Age was associated with FFQ validity for most of the studied dietary factors in women, whereas in men, the effect of age was less prominent. This finding recurred in betweenmethod correlation analyses performed in strata based on median age. Correlations for $\leq 57$ - and $>57$-year-old men differed only slightly, but appeared higher in $\leq 52$-year-old women compared with >52-year-olds (data not shown). In contrast, Paalanen et $a{ }^{(18)}$ found a poorer FFQ validity among young women (30-50 years) compared with older women (>50 years). Marks et al. ${ }^{(9)}$ did not observe an

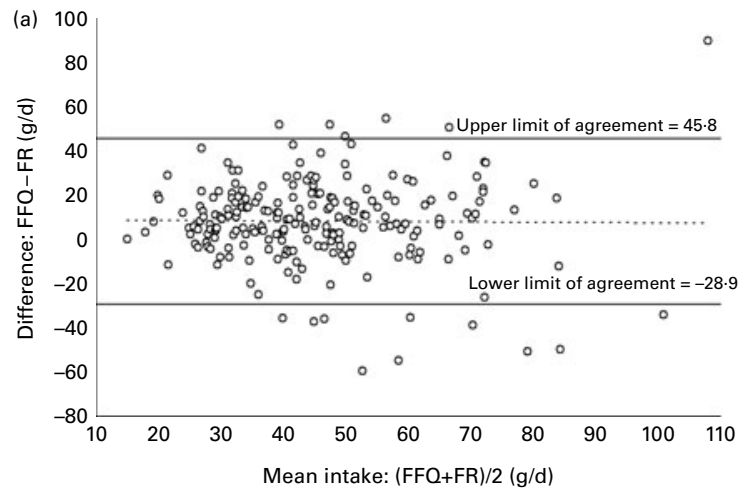

effect of age on FFQ validity in measuring carbohydrates in 25-75-year-old adults. Possible explanations for the present findings include that older age may undermine the subject's ability to diet reporting, which constitutes a demanding cognitive task ${ }^{(42)}$. General interest in health and food preparation can additionally predispose, especially women, to misreporting operating differently during the life course. The latter issue could also explain the few associations between education and FFQ validity evident in the present study. Earlier studies have, however, not shown such associations ${ }^{(9,19)}$

Earlier studies $^{(9,18)}$ have shown that BMI affects FFQ validity in measuring total carbohydrate, fibre and total sugars, but we did not. The present result remained as the linear regression analysis was repeated in age strata based on the median age cut-offs in both sexes. Additional between-method correlation analyses in median BMI-based strata revealed, however, that men in the lower BMI group $\left(<27 \mathrm{~kg} / \mathrm{m}^{2}\right)$ had slightly higher correlations than overweight/obese men $\left(\geq 27 \mathrm{~kg} / \mathrm{m}^{2}\right)$ (data not shown) similar to Paalanen et al. ${ }^{(18)}$. In women, the difference between normal weight $\left(<25 \mathrm{~kg} / \mathrm{m}^{2}\right)$ and overweight/obese $\left(\geq 25 \mathrm{~kg} / \mathrm{m}^{2}\right.$ ) did not appear prominently (data not shown). Regardless of BMI status in both sexes, correlations were acceptable and as in the present main analysis, lowest correlations in the overweight/obese men and women emerged for total sugars and dietary GI. This points

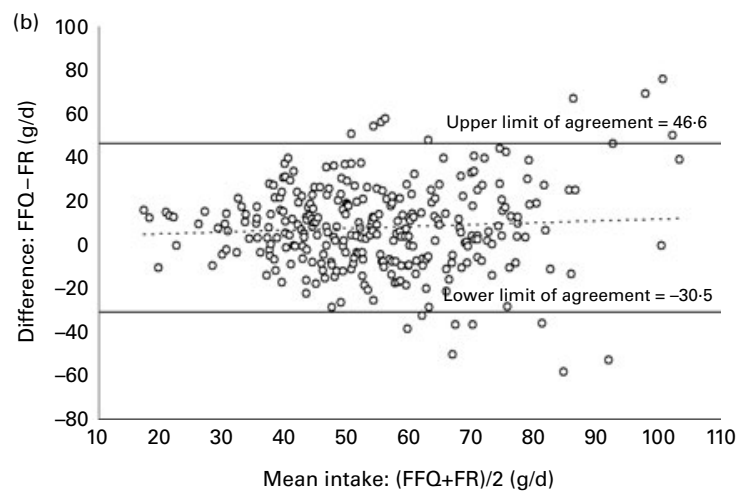

Fig. 2. Bland-Altman plots ${ }^{(31)}$ of sucrose from the FFQ and two $3 \mathrm{~d}$ food records (FR) for (a) men $(n 218 ; y=-0.016 \times+9.2, P=0.85)$ and (b) women $(n 292$; $y=-0.087 x+3.3, P=0.23)$. The linear trend $\left(\mathrm{H}_{0}\right.$ : slope $\left.=0, \alpha=0.05\right)$ and the limits of agreement are indicated. Intake values are energy-adjusted using the residual method $^{(30)}$. 
Table 3. Characteristics of study subjects associated with the difference in the energy-adjusted ${ }^{(30)}$ intakes of nutrients, dietary glycaemic index (GI) and the dietary glycaemic load (GL) from the FFQ and two $3 \mathrm{~d}$ food records (FR) (FFQ-FR)

\begin{tabular}{|c|c|c|c|c|c|c|c|c|}
\hline & \multicolumn{8}{|c|}{ Regression coefficient† } \\
\hline & \multicolumn{4}{|c|}{ Men $(n 218)$} & \multicolumn{4}{|c|}{ Women ( $n$ 292) } \\
\hline & \multirow[b]{2}{*}{ Age (years)§ } & \multicolumn{2}{|c|}{ Education $\ddagger$} & \multirow[b]{2}{*}{ BMI $\left(\mathrm{kg} / \mathrm{m}^{2}\right) \S$} & \multirow[b]{2}{*}{ Age (years)§ } & \multicolumn{2}{|c|}{ Education $\ddagger$} & \multirow[b]{2}{*}{ BMI $\left(\mathrm{kg} / \mathrm{m}^{2}\right) \S$} \\
\hline & & Low & Middle & & & Low & Middle & \\
\hline \multicolumn{9}{|c|}{ Nutrient/dietary factor difference (FFQ-FR) } \\
\hline Total carbohydrate $(\mathrm{g})$ & 0.312 & $2 \cdot 397$ & -0.894 & 0.370 & $0.589^{\star \star \star}$ & $-10 \cdot 548^{*}$ & -7.910 & 0.123 \\
\hline Starch $(g)$ & $0 \cdot 143$ & 2.528 & -0.104 & -0.120 & 0.046 & $-12 \cdot 077^{\star *}$ & $-2 \cdot 339$ & -0.011 \\
\hline Total sugars $(\mathrm{g})$ & 0.223 & -0.120 & 0.912 & 0.386 & $0.491^{\star *}$ & 1.548 & $-5 \cdot 345$ & 0.198 \\
\hline Fructose $(\mathrm{g})$ & -0.030 & 2.802 & 1.431 & 0.071 & $0.092^{*}$ & $2 \cdot 170$ & 0.493 & 0.046 \\
\hline Lactose $(\mathrm{g})$ & $-0.133^{*}$ & -2.755 & -2.831 & -0.235 & -0.020 & $-3 \cdot 208^{\star}$ & -2.255 & 0.088 \\
\hline Sucrose $(\mathrm{g})$ & 0.134 & -0.988 & $2 \cdot 346$ & 0.163 & $0 \cdot 244^{\star *}$ & 0.184 & -2.804 & -0.166 \\
\hline Dietary fibre $(\mathrm{g})$ & 0.010 & $-1 \cdot 001$ & $-2 \cdot 221^{*}$ & 0.021 & $0.064^{*}$ & -0.195 & 0.851 & 0.050 \\
\hline Insoluble dietary fibre (g) & -0.004 & -0.863 & $-1.648^{\star}$ & 0.001 & 0.035 & -0.473 & 0.360 & 0.036 \\
\hline Soluble polysaccharides (g) & 0.008 & -0.262 & -0.359 & 0.008 & $0.016^{*}$ & 0.119 & 0.065 & 0.005 \\
\hline Gl & 0.043 & 0.516 & 0.294 & 0.050 & $-0.043^{\star}$ & -0.778 & 0.799 & 0.003 \\
\hline $\mathrm{GL}$ & $0.325^{\star \star}$ & 1.676 & -0.209 & 0.323 & $0.249^{*}$ & $-8.965^{\star \star}$ & -2.990 & 0.094 \\
\hline
\end{tabular}

${ }^{\star} P<0.05 ;{ }^{\star \star} P<0.01 ;{ }^{* \star \star} P<0.0001$.

$\dagger$ In cases where the mean of intake (FFQ+FR/2) was significantly associated with the difference (FFQ-FR), the mean was included in the model (Table 2$)$.

¥ Reference: high education. Due to missing values, $n 217$ (for men) and $n 289$ (for women).

$\S$ Continuous variable.

towards a possible difficulty of overweight subjects to report certain food groups. The effect of this phenomenon on future studies cannot be ruled out.

Overall, reasons for the discrepancy of findings across studies relating to the effects of subject characteristics, including BMI, on FFQ validity are not well established. Given the small number of studies reporting these effects, more studies among different populations are needed. Most importantly, the effects are likely to be population- and FFQ-specific, further elucidating the need for FFQ validation studies and subsequent control for these factors in epidemiological analyses.

The present study is limited by the rather short time sequence of the FR, which may in part have led to the underestimation of FFQ validity. The similarity of consecutive FR days may hinder capturing the usual food consumption. Furthermore, the present study protocol did not allow for biomarkers reflecting carbohydrate fractions, such as the predictive biomarker for sugar intake ${ }^{(43)}$. This precludes the investigation of the measurement error, which inevitably accompanies the FFQ among other dietary assessment methods ${ }^{(44)}$. Major strengths of the present study include the large sample size ( $n$ 510) compared with validation studies in general (median $n 110)^{(14)}$. The subject distributions with regard to sex, age, education and BMI were similar to those of the entire study population ( $P$ values for differences $0 \cdot 13,0 \cdot 28,0.80$ and $0 \cdot 70$, respectively, data not shown), suggesting good representativeness. The present study also reports results for several carbohydrate fractions and dietary GI and GL and effects of subject characteristics on the relative FFQ validity, adding to the scant body of knowledge in these research fields.

In conclusion, the present study provides evidence that the updated 131-item FFQ is a reasonably good tool for measuring the intake of macronutrients and several carbohydrate fractions, as well as dietary GI and GL in epidemiological studies, in which analyses are based on subject ranking. Furthermore, the present study suggests that among Finnish adults, especially women, the relative FFQ validity is associated with the level of intake, age and, to a lesser extent, education. These factors should be considered in future by executing careful statistical adjustments in diet-disease relationship analysis, but also by striving towards further developing the FFQ method.

\section{Acknowledgements}

This study was supported by the Academy of Finland (111420, 118065 and 136895), the Finnish Cultural Foundation, the Juho Vainio Foundation and the Yrjö Jahnsson Foundation. The authors declare no personal or financial conflict of interest. N. E. K., L. M. V. and S. M. contributed to the original conception of this work. N. E. K. performed the statistical analyses in cooperation with H. T. and K. H. M. E. S. and L. M. V. contributed to the assignment of GI values to foods, which was carried out by N. E. K. H. R. contributed to the FFQ modifications. T. K. performed the calculation of nutrient intakes and dietary GI and GL. S. M., L. M. V., J. G. E. and M. P. participated in the design of the Dietary, Lifestyle and Genetic determinants of Obesity and Metabolic syndrome (DILGOM) study. N. E. K. wrote the manuscript and all co-authors critically appraised the paper.

\section{References}

1. DGAC (2010) Dietary Guidelines Advisory Committee report on dietary guidelines for Americans. Part D. Section 5: Carbohydrates. http://www.cnpp.usda.gov/DGAs2010-DGAC Report.htm (accessed September 2010). 
2. Mann J, Cummings JH, Englyst HN, et al. (2007) FAO/WHO scientific update on carbohydrates in human nutrition: conclusions. Eur J Clin Nutr 61, Suppl. 1, S132-S137.

3. Barclay AW, Petocz P, McMillan-Price J, et al. (2008) Glycemic index, glycemic load, and chronic disease risk - a meta-analysis of observational studies. Am J Clin Nutr 87, 627-637.

4. Cust AE, Slimani N, Kaaks R, et al. (2007) Dietary carbohydrates, glycemic index, glycemic load, and endometrial cancer risk within the European Prospective Investigation into Cancer and Nutrition cohort. Am J Epidemiol 166, 912-923.

5. Jakobsen MU, Dethlefsen C, Joensen AM, et al. (2010) Intake of carbohydrates compared with intake of saturated fatty acids and risk of myocardial infarction: importance of the glycemic index. Am J Clin Nutr 91, 1764-1768.

6. Barclay AW, Flood VM, Brand-Miller JC, et al. (2008) Validity of carbohydrate, glycaemic index and glycaemic load data obtained using a semi-quantitative food-frequency questionnaire. Public Health Nutr 11, 573-580.

7. Barrett JS \& Gibson PR (2010) Development and validation of a comprehensive semi-quantitative food frequency questionnaire that includes FODMAP intake and glycemic index. J Am Diet Assoc 110, 1469-1476.

8. Brunner E, Stallone D, Juneja M, et al. (2001) Dietary assessment in Whitehall II: comparison of $7 \mathrm{~d}$ diet diary and foodfrequency questionnaire and validity against biomarkers. $\mathrm{BrJ}$ Nutr 86, 405-414.

9. Marks GC, Hughes MC \& van der Pols JC (2006) The effect of personal characteristics on the validity of nutrient intake estimates using a food-frequency questionnaire. Public Health Nutr 9, 394-402.

10. Männistö S, Virtanen M, Mikkonen T, et al. (1996) Reproducibility and validity of a food frequency questionnaire in a case-control study on breast cancer. J Clin Epidemiol 49, 401-409.

11. Pietinen P, Hartman AM, Haapa E, et al. (1988) Reproducibility and validity of dietary assessment instruments. I. A self-administered food use questionnaire with a portion size picture booklet. Am J Epidemiol 128, 655-666.

12. Willett W (1998) Nutritional Epidemiology, 2nd ed. New York: Oxford University Press.

13. Bingham SA, Cassidy A, Cole TJ, et al. (1995) Validation of weighed records and other methods of dietary assessment using the $24 \mathrm{~h}$ urine nitrogen technique and other biological markers. BrJ Nutr 73, 531-550.

14. Cade J, Thompson R, Burley V, et al. (2002) Development, validation and utilisation of food-frequency questionnaires - a review. Public Health Nutr 5, 567-587.

15. Molag ML, de Vries JH, Ocké MC, et al. (2007) Design characteristics of food frequency questionnaires in relation to their validity. Am J Epidemiol 166, 1468-1478.

16. Willett WC, Sampson L, Stampfer MJ, et al. (1985) Reproducibility and validity of a semiquantitative food frequency questionnaire. Am J Epidemiol 122, 51-65.

17. Block G \& Hartman AM (1989) Issues in reproducibility and validity of dietary studies. Am J Clin Nutr 50, 1133-1138, discussion 1231-1135.

18. Paalanen L, Männistö S, Virtanen MJ, et al. (2006) Validity of a food frequency questionnaire varied by age and body mass index. J Clin Epidemiol 59, 994-1001.

19. Longnecker MP, Lissner L, Holden JM, et al. (1993) The reproducibility and validity of a self-administered semiquantitative food frequency questionnaire in subjects from South Dakota and Wyoming. Epidemiology 4, 356-365.
20. Vartiainen E, Laatikainen T, Peltonen M, et al. (2010) Thirty-five-year trends in cardiovascular risk factors in Finland. Int J Epidemiol 39, 504-518.

21. Paturi M, Tapanainen $\mathrm{H}$, Reinivuo $\mathrm{H}$, et al. (2008) The National FINDIET 2007 Survey (in Finnish, Tables, Figures and Summary in English). Publications of the National Public Health Institute B23/2008. Helsinki: National Public Health Institute. http://www.ktl.fi/attachments/suomi/ julkaisut/julkaisusarja_b/2008/2008b23.pdf

22. Paturi M, Nieminen R, Reinivuo H, et al. (2006) Picture Book of Food Portion Sizes (in Finnish, Food Names and Summary in English). Publications of the National Public Health Institute B11/2006. Helsinki: National Public Health Institute. http://www.ktl.fi/attachments/suomi/julkaisut/julkaisusarja_b/2006/2006b11.pdf

23. Reinivuo H, Hirvonen T, Ovaskainen ML, et al. (2010) Dietary survey methodology of FINDIET 2007 with a risk assessment perspective. Public Health Nutr 13, 915-919.

24. Kaartinen NE, Similä ME, Pakkala H, et al. (2010) Glycaemic index values in the Finnish food composition database: an approach to standardised value documentation. Eur J Clin Nutr 64, Suppl. 3, S68-S72.

25. Similä ME, Valsta LM, Virtanen MJ, et al. (2009) Glycaemic index database for the epidemiological Alpha-Tocopherol, Beta-Carotene Cancer Prevention (ATBC) Study. Br J Nutr 101, 1400-1405.

26. Wolever TM, Jenkins DJ, Jenkins AL, et al. (1991) The glycemic index: methodology and clinical implications. Am J Clin Nutr 54, 846-854.

27. Wolever TM, Nguyen PM, Chiasson JL, et al. (1994) Determinants of diet glycemic index calculated retrospectively from diet records of 342 individuals with non-insulin-dependent diabetes mellitus. Am J Clin Nutr 59, 1265-1269.

28. Salmerón J, Ascherio A, Rimm EB, et al. (1997) Dietary fiber, glycemic load, and risk of NIDDM in men. Diabetes Care 20, $545-550$.

29. Venn BJ \& Green TJ (2007) Glycemic index and glycemic load: measurement issues and their effect on diet-disease relationships. Eur J Clin Nutr 61, Suppl. 1, S122-S131.

30. Willett W \& Stampfer MJ (1986) Total energy intake: implications for epidemiologic analyses. Am J Epidemiol 124, 17-27.

31. Bland JM \& Altman DG (1986) Statistical methods for assessing agreement between two methods of clinical measurement. Lancet i, 307-310.

32. Serra-Majem L, Frost Andersen L, Henrique-Sanchez $\mathrm{P}$, et al. (2009) Evaluating the quality of dietary intake validation studies. Br J Nutr 102, Suppl. 1, S3-S9.

33. McKeown NM, Day NE, Welch AA, et al. (2001) Use of biological markers to validate self-reported dietary intake in a random sample of the European Prospective Investigation into Cancer United Kingdom Norfolk cohort. Am J Clin Nutr 74, 188-196.

34. Riboli E, Elmstahl S, Saracci R, et al. (1997) The Malmo Food Study: validity of two dietary assessment methods for measuring nutrient intake. Int J Epidemiol 26, Suppl. 1, S161-S173.

35. Friis S, Kruger Kjaer S, Stripp C, et al. (1997) Reproducibility and relative validity of a self-administered semiquantitative food frequency questionnaire applied to younger women. J Clin Epidemiol 50, 303-311.

36. Tjønneland A, Overvad K, Haraldsdottir J, et al. (1991) Validation of a semiquantitative food frequency questionnaire developed in Denmark. Int J Epidemiol 20, 906-912.

37. Du H, van der AD, van Bakel MM, et al. (2009) Reproducibility and relative validity of dietary glycaemic index and 
glycaemic load assessed by the food-frequency questionnaire used in the Dutch cohorts of the European Prospective Investigation into Cancer and Nutrition. Br J Nutr 102, 601-604.

38. Levitan EB, Westgren CW, Liu S, et al. (2007) Reproducibility and validity of dietary glycemic index, dietary glycemic load, and total carbohydrate intake in 141 Swedish men. Am J Clin Nutr 85, 548-553.

39. Murakami K, Sasaki S, Takahashi Y, et al. (2008) Reproducibility and relative validity of dietary glycaemic index and load assessed with a self-administered diet-history questionnaire in Japanese adults. Br J Nutr 99, 639-648.

40. Poslusna K, Ruprich J, de Vries JH, et al. (2009) Misreporting of energy and micronutrient intake estimated by food records and 24 hour recalls, control and adjustment methods in practice. Br J Nutr 101, Suppl. 2, S73-S85.
41. Maurer J, Taren DL, Teixeira PJ, et al. (2006) The psychosocial and behavioral characteristics related to energy misreporting. Nutr Rev 64, 53-66.

42. McNeill G, Winter J \& Jia X (2009) Diet and cognitive function in later life: a challenge for nutrition epidemiology. Eur J Clin Nutr 63, Suppl. 1, S33-S37.

43. Tasevska N, Midthune D, Potischman N, et al. (2011) Use of the predictive sugars biomarker to evaluate self-reported total sugars intake in the Observing Protein and Energy Nutrition (OPEN) study. Cancer Epidemiol Biomarkers Prev 20, 490-500.

44. Schatzkin A, Subar AF, Moore S, et al. (2009) Observational epidemiologic studies of nutrition and cancer: the next generation (with better observation). Cancer Epidemiol Biomarkers Prev 18, 1026-1032.

Appendix. Cross-classification of crude and energy-adjusted nutrient, glycaemic index (GI) and glycaemic load (GL) distribution quintiles from the FFQ and two $3 \mathrm{~d}$ food records for men and women

\begin{tabular}{|c|c|c|c|c|c|c|c|c|}
\hline & \multicolumn{4}{|c|}{ Men $(n 218)$} & \multicolumn{4}{|c|}{ Women (n 292) } \\
\hline & \multicolumn{2}{|c|}{ Same \pm 1 quintile (\%) } & \multicolumn{2}{|c|}{ Opposite quintile (\%)† } & \multicolumn{2}{|c|}{ Same \pm 1 quintile (\%) } & \multicolumn{2}{|c|}{ Opposite quintile (\%)† } \\
\hline & Crude & Energy-adjusted $\ddagger$ & Crude & Energy-adjusted $\ddagger$ & Crude & Energy-adjustedł & Crude & Energy-adjusted $\neq$ \\
\hline \multicolumn{9}{|l|}{ Nutrient/dietary factor } \\
\hline Energy $(\mathrm{kJ})$ & 65 & - & $2 \cdot 8$ & - & 58 & - & $6 \cdot 5$ & - \\
\hline Total carbohydrate (g) & 68 & 70 & $2 \cdot 8$ & 1.8 & 60 & 75 & $2 \cdot 7$ & $2 \cdot 1$ \\
\hline Starch $(g)$ & 67 & 67 & $2 \cdot 3$ & $2 \cdot 3$ & 65 & 72 & 3.8 & 1.7 \\
\hline Total sugars (g) & 64 & 62 & $3 \cdot 2$ & 5.5 & 64 & 64 & $4 \cdot 1$ & $3 \cdot 1$ \\
\hline Fructose (g) & 65 & 73 & $3 \cdot 2$ & $3 \cdot 2$ & 60 & 70 & $3 \cdot 8$ & $3 \cdot 8$ \\
\hline Lactose $(\mathrm{g})$ & 81 & 83 & 0.0 & 0.5 & 81 & 83 & 1.0 & 1.0 \\
\hline Sucrose (g) & 70 & 77 & $2 \cdot 3$ & 3.7 & 66 & 70 & 3.4 & $2 \cdot 4$ \\
\hline Dietary fibre $(\mathrm{g})$ & 79 & 80 & 0.5 & 0.9 & 68 & 76 & $3 \cdot 1$ & $1 \cdot 0$ \\
\hline Insoluble dietary fibre (g) & 78 & 78 & 0.9 & 1.4 & 68 & 78 & $2 \cdot 7$ & 1.0 \\
\hline Soluble polysaccharides (g) & 79 & 82 & 1.4 & 1.4 & 65 & 76 & $2 \cdot 4$ & 1.4 \\
\hline GI & 62 & 65 & $2 \cdot 3$ & $4 \cdot 1$ & 69 & 69 & 3.1 & $3 \cdot 1$ \\
\hline $\mathrm{GL}$ & 68 & 69 & $3 \cdot 2$ & 1.0 & 58 & 72 & $2 \cdot 7$ & 1.4 \\
\hline
\end{tabular}

† Disagreement by four quintiles (gross misclassification).

‡ Energy-adjusted using the residual method ${ }^{(30)}$. 